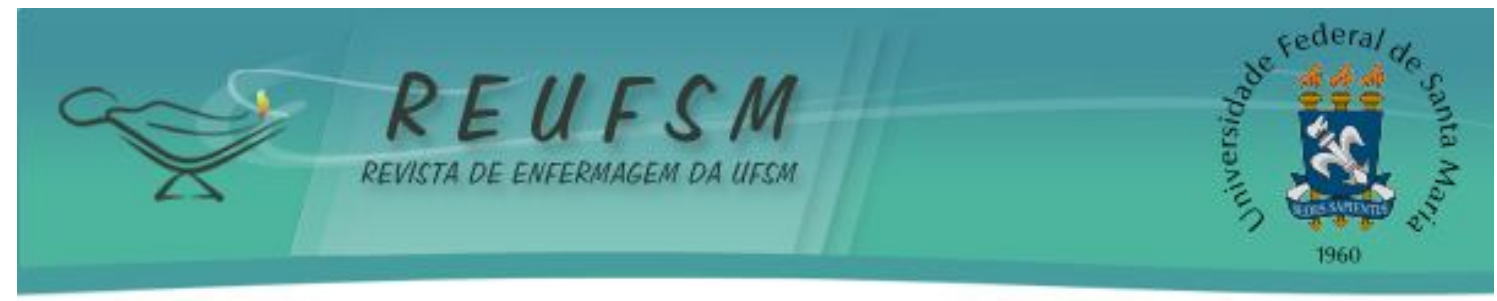

ARTIGO DE REVISÃO

\title{
EXPERIÊNCIAS DE SISTEMATIZAÇÃO DA ASSISTÊNCIA DE ENFERMAGEM NO BRASIL: UM ESTUDO BIBLIOGRÁFICO
}

\section{EXPERIENCES CARE SYSTEM NURSING IN BRAZIL: A BIBLIOGRAPHIC STUDY \\ EXPERIENCIAS DE SISTEMATIZACIÓN DE LA ASISTENCIA DE ENFERMERÍA EN BRASIL: UN EST UDIO BIBLIOGRÁFICO}

\author{
Ricardo Bezerra Cavalcante ${ }^{1}$ \\ Alba Otoni ${ }^{2}$ \\ Mariana Ferreira Vaz Gontijo Bernardes ${ }^{3}$ \\ Simone Graziele Silva Cunha ${ }^{4}$ \\ Camila da Silveira Santos ${ }^{5}$ \\ Poliana Cavalcante da Silva ${ }^{6}$
}

RESUMO: Buscou-se identificar experiências de Sistematização da Assistência de Enfermagem (SAE) na rotina diária de trabalho da enfermagem em instituições brasileiras. Realizou-se busca eletrônica na Biblioteca Virtual de Saúde nas bases "Scielo, Lilacs e Bdenf" por meio das palavras "Sistematização Enfermagem" e "Processo Enfermagem". Selecionaram-se trabalhos de campo realizados em instituições nacionais no período entre 1979 e 2009, totalizando 71 estudos. As principais temáticas foram: estudo de caso $28 \%$ elaboração de instrumento de coleta $21 \%$ e perfil de diagnósticos de enfermagem 20\% Apenas 32\% demonstraram a aplicação das cinco etapas do Processo de Enfermagem. Predominam experiências na atenção hospitalar 92\% As principais teorias de enfermagem foram: Necessidades Humanas Básicas 36\% e Déficit do Auto Cuidado 17\% Conclui-se que apesar da resolução do COFEN determinando a obrigatoriedade da SAE em todas as instituições de saúde brasileiras, a implantação e manutenção dessa metodologia ainda se desenvolvem de forma lenta e gradativa.

Descritores: Enfermagem; Assistência de enfermagem; Diagnóstico de enfermagem.

ABST RACT: We sought to identify experiences of Nursing Care System (NCS) in the daily routine of nursing work in Brazilian institutions. Electronic search was conducted in the Virtual Health Library on the ground "SciELO, LILACS and BDENF" by of words "Systematization Nursing" and "Nursing Process". Were selected field work carried out in national institutions in the period between 1979 and 2009, totaling 71 studies. The main themes were: 28\% case study, preparation of collection instrument profile of $21 \%$ and $20 \%$ nursing diagnoses. Only $32 \%$ demonstrated the application of the five steps of nursing process. The predominant experience in hospital care $92 \%$ The main theories of nursing were: $36 \%$ Basic Human Needs and Self Care Deficit of $17 \%$ We conclude that despite the resolution of COFEN determining the mandatory in all SAE Brazilian health institutions, implementation and maintenance of this methodology is still developing slowly and gradually.

Descriptors: Nursing; Nursing care; Nursing diagnosis.

\footnotetext{
1 Enfermeiro. Prof. Curso de Enfermagem da Universidade Federal de São João Del Rei. E-mail: ricardocavalcanteufmg@yahoo.com.br

2 Enfermeira. Profạ. Curso de Enfermagem da Universidade Federal de São João Del Rei. E-mail: albaotoni01@yahoo.com.br

3 Acadêmica de Enfermagem da Universidade Federal de São João Del Rei. E-mail: marianagontij oufsj@gmail.com

${ }_{5}^{4}$ Acadêmica de Enfermagem da Universidade Federal de São J oão Del Rei. E-mail: simonegraziele@ig.com.br

5 Acadêmica de Enfermagem da Universidade Federal de São João Del Rei. E-mail: camilasilveiraufsj@gmail.com

${ }^{6}$ Enfermeira. Orientadora de Estágios do Curso técnico de Enfermagem do Hospital São J oão de Deus. E-mail: polianacavalcantes@yahoo.com.br
} 


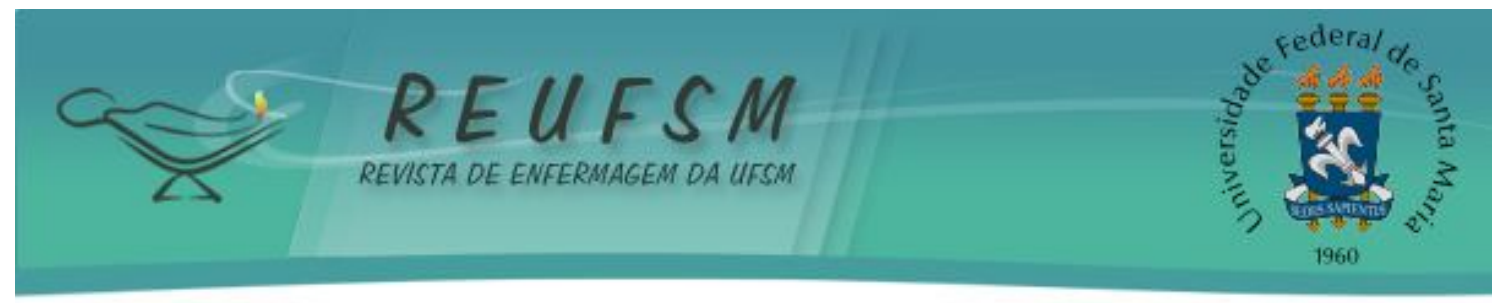

RESUMEN: Este artículo buscó identificar las experiencias del Sistematización de Cuidados de Enfermería, en la rutina diaria de trabajo de enfermería en las instituciones brasileñas. Búsqueda el ectrónica se realizó en la Biblioteca Virtual de Salud en la base ", SciELO, LILACS y BDENF" por de las palabras "Sistematización de Enfermería" y "Proceso de Enfermería". El trabajo se han seleccionado de campo realizado en las instituciones nacionales en el período comprendido entre 1979 y 2009, un total de 71 estudios. Los principales temas fueron: $28 \%$ caso de estudio, la preparación del perfil de colección de instrumentos de $21 \%$ y $20 \%$ diagnósticos de enfermería. Sólo el 32\% de manifiesto la aplicación de los cinco pasos del proceso de enfermería. La experiencia predominante en la atención hospitalaria del $92 \%$ Las principales teorías de enfermería: un 36\% las necesidades humanas básicas y déficit de autocuidado del 17\% Llegamos a la conclusión que a pesar de la exigencia de SAE en todas las instituciones de salud brasileño, a implementación y mantenimiento de esta metodología aún se está desarrollando lentamente y gradual.

Descriptores: Enfermería; Cuidados de enfermería; Diagnóstico de enfermería.

\section{INTRODUÇÃO}

Durante a década de 70 observou-se um movimento mundial dos profissionais enfermeiros na direção de organizar e o planejar a assistência de enfermagem baseada na cientificidade do Processo de Enfermagem (PE). No Brasil, a partir dos estudos de Wanda de Aguiar Horta, uma das pioneiras a refletir sobre o Processo de Enfermagem, iniciou-se, nessa mesma época, um apontamento para a necessidade de se introduzir a Sistematização da Assistência de Enfermagem (SAE) nas instituições de saúde brasileiras. ${ }^{1}$

A SAE consiste em uma metodologia para organizar e sistematizar o cuidado com base no conhecimento científico, permitindo ao enfermeiro a aplicação desses na identificação das necessidades de cuidados de enfermagem, além da promoção de maior segurança e qualidade durante a assistência prestada. Ressalta-se ainda que essa metodologia de assistência proporciona autonomia ao enfermeiro a partir da definição do corpo de conhecimentos e técnicas necessárias à prática assistencial de excelência. ${ }^{2}$

Segundo a resolução 358\2009 do Conselho Federal de Enfermagem, o enfermeiro deverá realizar o Processo de Enfermagem (PE), constituído de cinco etapas, sendo elas: Coleta de dados ou Histórico de Enfermagem, Diagnóstico de enfermagem, Planejamento, Implementação e Avaliação. Afirma ainda que o PE deverá ser realizado em todas as instituições de saúde públicas ou privadas, de modo deliberativo e sistemático, baseandose em um suporte teórico que oriente as etapas do processo. ${ }^{3}$

A profissão de enfermagem vem se constituindo como um componente essencial na qualidade em saúde, acompanhando assim mudanças nas relações interpessoais, sociais, políticas, no campo tecnológico e no modelo das organizações dos serviços. ${ }^{4}$ A SAE contribui para a expansão do conhecimento, a qualidade da assistência e melhores registros das informações de enfermagem. ${ }^{5}$

A primeira resolução do COFEN que determinou a obrigatoriedade da SAE em todo território nacional foi publicada no ano de 2002, sendo posteriormente substituída pela 358/2009. Nove anos depois do primeiro movimento de legalização da SAE se faz necessário visualizar o panorama geral em que as experiências de sistematização da assistência de enfermagem vêm ocorrendo.

Nesta perspectiva, o estudo proposto tem o objetivo principal de identificar as experiências de Sistematização da Assistência de Enfermagem desenvolvidas em instituições de saúde brasileiras. Os questionamentos que nortearam este estudo foram: "Como as experiências de sistematização da assistência de enfermagem vêm ocorrendo no Brasil ao longo dos anos? Quais são as principais temáticas discutidas nas experiências publicadas? Quais são as principais teorias utilizadas para embasar o PE? Como ocorre a distribuição das experiências entre os níveis assistenciais primário, secundário e terciário? Quais são os setores das instituições 


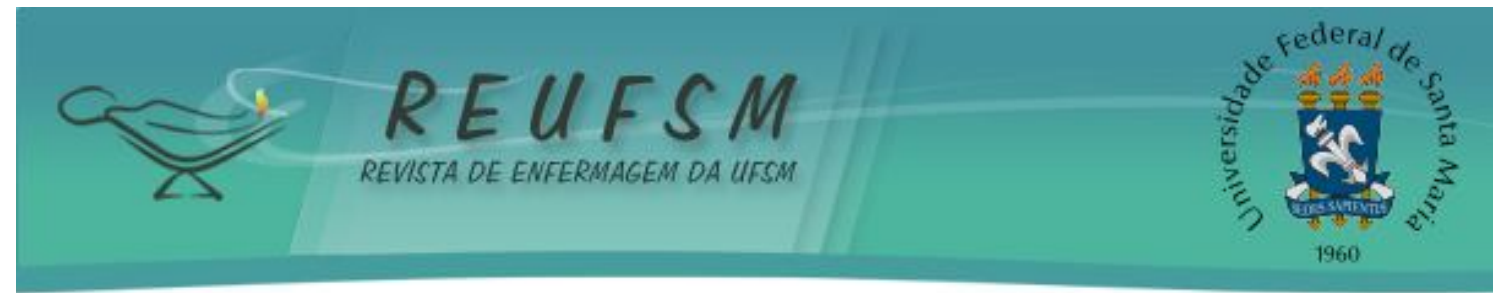

de saúde onde predominam as experiências de sistematização da assistência de enfermagem? E por fim, as cinco etapas do PE estão sendo plenamente implantadas?".

Espera-se que este estudo bibliográfico possa responder a estes questionamentos e promova um debate sobre a importância da SAE, suas evoluções, bem como a necessidade de novos avanços.

\section{MÉTODO}

Trata-se de um estudo exploratório, descritivo com abordagem quantitativa. Para tanto, optou-se pelo estudo bibliográfico, onde se utilizou as bases de dados "Scielo, Bdenf e Lilacs" a partir da Biblioteca Virtual de Saúde (BVS). As palavras utilizadas para busca foram "Sistematização Enfermagem" e "Processo Enfermagem". Criou-se um banco de dados, onde foram definidos previamente os seguintes itens: ano de publicação do estudo, temática principal do estudo, nível assistencial onde a SAE foi aplicada, setor da instituição onde a experiência se desenvolveu, teorias utilizadas para direcionar o PE e as fases do PE implantadas. Estes itens foram definidos a partir dos questionamentos de pesquisa que nortearam a investigação.

A partir da leitura dos resumos, foram selecionados estudos que atendiam os seguintes critérios: publicações que apresentavam as experiências de implantação e/ ou avaliação da $\mathrm{SAE}$, em instituições de saúde brasileiras, no período de 1979 a 2009 . Foram excluídos os trabalhos de reflexão teórica e revisão bibliográfica que tratavam do tema, pois se desejou captar apenas as experiências de aplicação da SAE em alguma instituição. Após a coleta, os dados foram armazenados em um banco de dados desenvolvido no "Microsoft Excel", onde se procedeu a análise estatística descritiva.

\section{RESULTADOS E DISCUSSÕES}

\section{Evolução histórica das experiências de SAE no Brasil}

No gráfico 1 observa-se que a SAE vem sendo discutida, no Brasil, a partir do final da década de 70 até os dias atuais. No entanto, percebe-se que os anos 70 e 80 foram marcados por uma constância e um pequeno número de publicações abordando a temática. Nos anos 90 e 2000 visualiza-se um incremento significativo no número de publicações, o que pode demonstrar a relevância da temática no período. Interessantemente observou-se uma queda importante no número de publicações referentes a esse tema no ano 2002 que foi 0 ano de legalização da implantação da SAE em instituições de Saúde Brasileiras, somente voltando a recuperar os números de produção dois anos mais tarde. Desde então, a produção científica indexada sobre o assunto vem oscilando com tendências ao crescimento até os dias atuais.

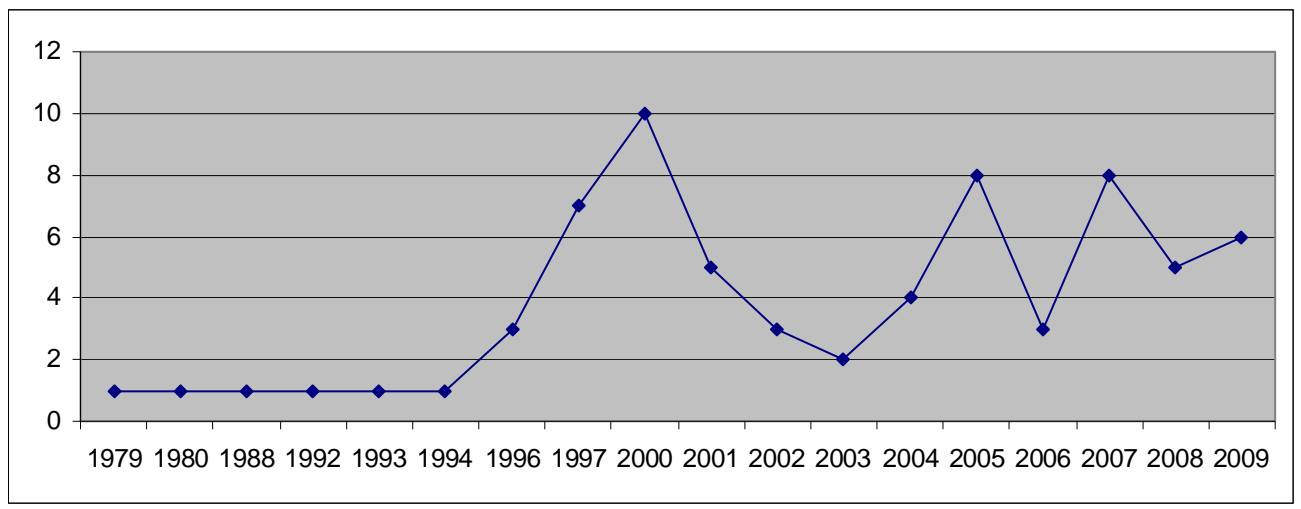

Gráfico 1 - Experiências de SAE publicadas em revistas indexadas no Brasil - (1979 - 2009). 


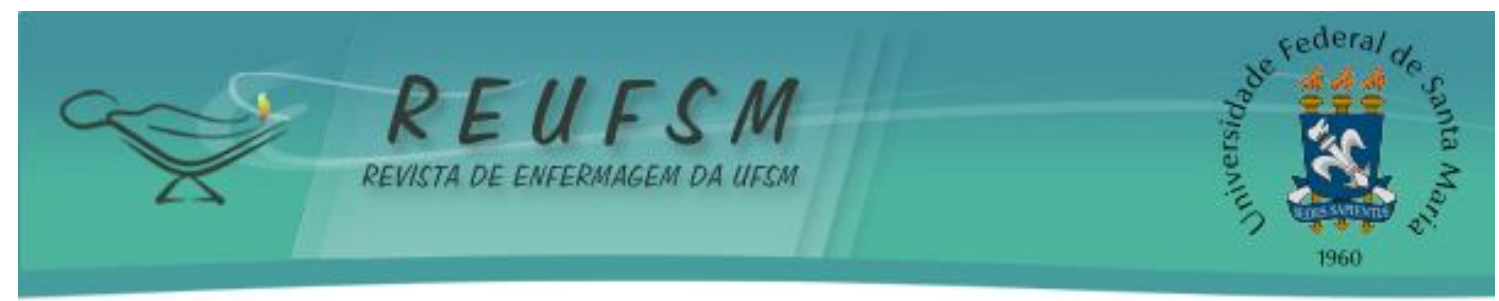

Apesar do crescimento do número de publicações sobre a temática ao longo dos anos, percebese que as décadas de 60, 70, 80 e meados da década de 90 possuem publicações ainda incipientes. Neste período, ressaltam-se as discussões da enfermeira e teórica "Wanda de Aguiar Horta" que se destacou com seu pioneirismo por apresentar aos enfermeiros brasileiros a possibilidade de aplicação do PE na sua prática diária de trabalho. A autora em 1963 apresenta suas primeiras reflexões introduzindo-as em disciplinas optativas de universidades paulistas. Em 1965 o plano de cuidados de enfermagem já era recomendado pelo Congresso Brasileiro de Enfermagem. Porém não fora assumido como prioridade pelos gestores de serviços de saúde como uma função prioritária do profissional. ${ }^{6}$

As primeiras experiências de SAE publicadas, nas décadas de 70 e 80 , em revistas indexadas brasil eiras são originárias de docentes de enfermagem relatando suas experiências em hospitais-escola. Isto pode evidenciar que a grande maioria das experiências de aplicação da $\mathrm{SAE}$, neste período, ainda estavam vinculadas ao contexto acadêmico. Esta situação apontou ainda para a necessidade de evoluções no estabelecimento efetivo da SAE nas instituições de saúde espalhadas pelo território nacional.

A partir da metade da década de 90 as experiências de aplicação da SAE nas instituições de saúde ganham força, segundo as publicações daquele período, e começam a se multiplicar atingindo o seu ápice nos anos 2000. Essa ascensão na opção pela aplicação da SAE parece ser resultado de uma efervescência nas reflexões sobre a temática, não só no Brasil, como em todo o mundo.,8

Em 2002 ressalta-se o marco regulatório acerca da implantação e manutenção da SAE no Brasil. Este marco foi definido pela resolução do COFEN 272/2002 que define a obrigatoriedade, até 0 ano de 2012, da implantação do PE em todas as instituições públicas e privadas, de forma organizada e sistemática, baseando-se em uma teoria que possa nortear as etapas do PE. ${ }^{9}$ A partir da publicação da resolução referida, embora se tenha um registro inicial da queda de publicações, na sequência houve um aumento no número de experiências realizadas e publicadas, mesmo diante de oscilações importantes até 0 ano de 2009.

A falta de constância na elevação no número de publicações nos leva a questionar se quando caem os números de publicações significa que houve, de fato, uma queda na implementação da SAE nas instituições de saúde ou se, simplesmente não se investiu em publicações de experiências naquele período.

Em 2009, o Conselho Federal de Enfermagem publica a resolução 358/ 2009 que endossa a obrigatoriedade da implantação da SAE em todo território nacional, destaca a necessidade iminente da utilização das teorias de enfermagem como base para a operacionalização do PE, além de reforçar a importância do registro de todo o processo no prontuário do paciente. ${ }^{3}$

Apesar da constatação da evolução do número de publicações das experiências de SAE em instituições de saúde brasileiras, percebe-se que este incremento vem ocorrendo de forma lenta e essencialmente atrelado a grupo de pesquisas e trabalhos desenvolvidos dentro das universidades. ${ }^{6}$ Como já destacado, este fato talvez esteja ligado à possibilidade de aplicação da SAE em instituições que não tenham sido publicadas em revistas nacionais indexadas. Entretanto, se esse fato se confirmar é preciso alertar os enfermeiros assistenciais da importância do avanço destas publicações, a fim de promover 0 conhecimento destas experiências, bem como as diversas possibilidades de aplicação da SAE.

\section{As principais temáticas relacionadas com as experiências}

No que se refere às temáticas retratadas nos estudos, observou-se que do total de 71 trabalhos publicados, a maioria $28 \%(20)$ apresentava estudos de caso de um determinado paciente onde se aplicou pelo menos uma das etapas do PE. Posteriormente, observou-se que $21 \%$ (15) abordavam a elaboração de um instrumento de coleta de dados, focando apenas a primeira etapa do processo de enfermagem. Os outros $20 \%$ (14) dos estudos trabalharam o perfil de diagnósticos de enfermagem de pacientes em setores específicos de instituições de saúde. 


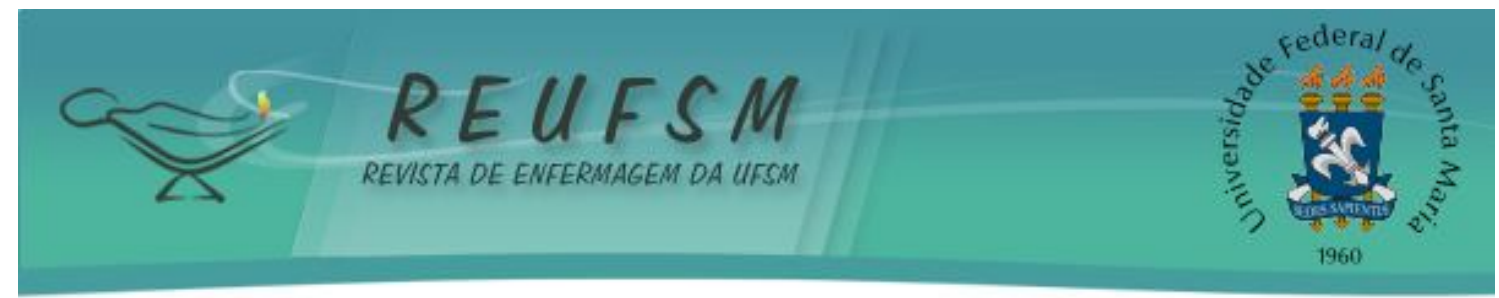

Ainda, 17\% (12) das publicações abordavam a aplicação do PE, mesmo que parcialmente, na assistência a pacientes em instituições brasileiras. Os demais trabalhos apresentados na tabela 1 abordavam os seguintes temas: análise da implantação da SAE 3\% (2), aplicações das classificações de Enfermagem 3\%(2), consulta de enfermagem sistematizada 3\%(2), aplicação da Sistematização da Assistência de Enfermagem Perioperatória (SAEP) 1\%(1), aplicação e avaliação dos conceitos da Teoria de Orem 1\%1), informatização do processo de enfermagem $1 \% 1$ ) e perfil de diagnóstico em conjunto com as possíveis intervenções de enfermagem $1 \% 1$ ).

A revisão dessas publicações demonstra que existe uma ampla dimensão de discussão acerca do tema SAE, contudo, essa diversidade não mostra uma ef etividade na implementação dessa metodologia de assistência, pois, o número de trabalhos demonstrando a aplicação do PE em sua totalidade, com todas as suas cinco etapas, ainda são incipientes (Tabela 2).

Tabela 1 - Fases do Processo de Enfermagem

\begin{tabular}{lcc}
\multicolumn{1}{c}{ Fases do PE } & N & \% \\
\hline Todas as fases do PE implantadas & 23 & $32 \%$ \\
Apenas al gumas fases do PE implantadas & 36 & $\mathbf{5 1 \%}$ \\
Não definido & 12 & $17 \%$ \\
\hline TOTAL & 71 & $100 \%$ \\
\hline
\end{tabular}

A maioria dos trabalhos publicados 51\% (36) apresentam experiências onde houve a aplicação parcial do PE. Apenas $32 \%(21)$ das experiências demonstram a aplicação do PE com todas as suas fases, de acordo com a resolução do COFEN 358/2009. Já 17\% (12) dos estudos não definiram no trabalho qual fase do Processo de Enfermagem se encontra a instituição apresentada no estudo. Assim, os dados encontrados nos remetem a uma antiga discussão em enfermagem, "estamos publicando as nossas experiências? Ou não estamos trabalhando com embasamento científico?" Acredita-se que de fato houve evolução nas vivências a partir de uma assistência balizada por preceitos científicos, mas ainda nos falta a socialização, por meio de publicações, de experiências profissionais nas quais o PE tenha sido aplicado com todas as cinco etapas previstas na resolução vigente.

O PE implementado na sua totalidade direciona atitudes sistematizadas e interrelacionadas com fins a assistência de qualidade ao ser humano, guiando as decisões necessárias para um cuidado de excelência da enfermagem, na concepção aprimorada do cuidado, na conjuntura familiar e comunitária, e não apenas orienta um cuidado focado na doença. ${ }^{21}$ Desta forma, é imprescindível que o PE seja aplicado em sua totalidade, perpassando pelas cinco fases inter-relacionadas, direcionando as devidas intervenções para o alcance do objetivo final que é uma maior qualidade e segurança na assistência de enfermagem. ${ }^{22}$ O PE quando aplicado de forma parcial fragiliza a integração das etapas, fragmentando as informações e consequentemente o cuidado. Isto pode proporcionar 0 risco de uma assistência insuficiente. Desta forma, mais uma vez se afirma a necessidade de aperfeiçoar o PE como uma metodologia incorporada à rotina de trabalho da equipe de enfermagem, bem como a forma sistemática de atuação profissional.

Muitas são as dificuldades registradas que podem estar contribuindo para a ineficiência da aplicação total do PE no dia a dia da assistência de enfermagem. Alguns autores apontam que as dificuldades para a implementação da PE não se restringem apenas à prática profissional, mas parecem se iniciar desde o ensino acadêmico quando há uma dificuldade, por parte dos docentes, de desenvolverem estratégicas didáticas padronizadas e eficientes para 0 aprendizado efetivo dessa metodologia de assistência. ${ }^{23,24}$ 


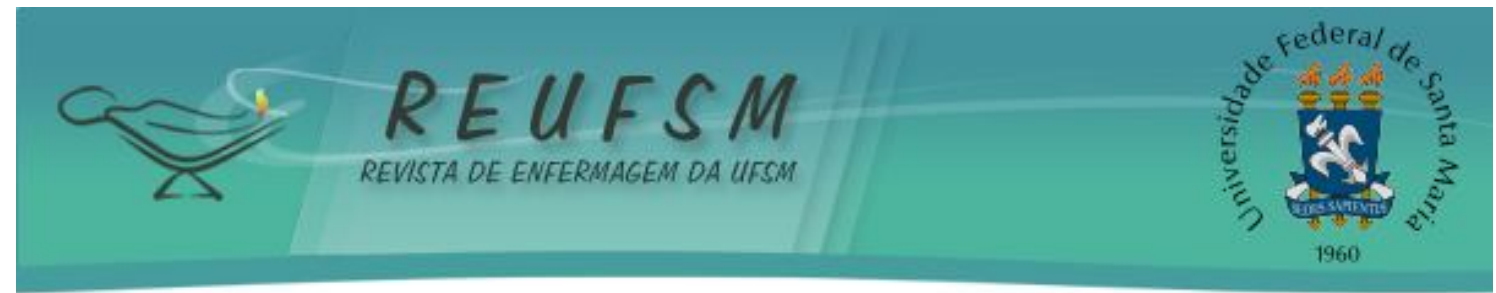

Ainda indicam que as maiores dificuldades relativas à operacionalização da SAE são: complexidade do PE, a falta de uniformidade nas etapas dificultando a compreensão dos profissionais, a falta de conhecimento efetivo sobre o tema e por conseqüência a falta de um marco conceitual não somente entre os profissionais da instituição de saúde como também entre os docentes de uma mesma instituição de ensino. ${ }^{23,24} \mathrm{E}$ por fim, cita-se a inércia dos órgãos fiscalizadores, em alguns estados, no sentido de realizarem uma fiscalização eficaz o suficiente para fazer cumprir a resolução 358\2009.

\section{Níveis de assistência à saúde onde as experiências foram realizadas e setores das instituições onde foram desenvolvidas.}

Através da análise dos trabalhos identificou-se que as experiências de SAE implantadas no Brasil estão voltadas para o nível secundário e terciário, mais especificamente na área hospitalar, representando um total de $92 \%$ (65). Apesar da existência de publicações sobre a aplicação da SAE seja na saúde coletiva, identificou-se que no nível primário a SAE é pouco divulgada, sendo registrado apenas 4\%(3) das publicações sobre SAE em serviços de atenção primária. As demais experiências ocorreram em Instituições de longa permanência representando 3\%(2) e serviços de remoção aeromédica 1\%1) (Gráfico 2).

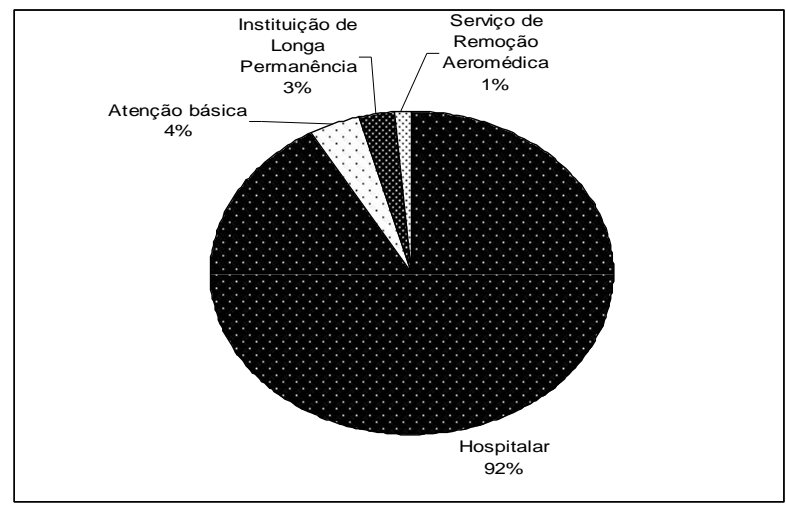

Gráfico 2 - Níveis assistenciais onde as experiências se desenvolveram

Destaca-se a resolução 358/2009 do COFEN que aponta a obrigatoriedade da implantação da SAE em todo o território nacional, bem como em todos os níveis assistenciais, especialidades e setores das instituições de saúde. No entanto, percebe-se a concentração dos estudos publicados relacionados ao nível secundário e terciário de assistência a saúde, focados principalmente na área hospitalar, ficando a atenção básica (nível primário) subjugada a um pequeno número de experiências publicadas.

Neste sentido, resgata-se que a mudança do modelo assistencial hospitalocêntrico para atenção básica desde 1994 vem sendo prioridade do Ministério da Saúde. O Programa de Saúde da Família (PSF) vem consolidar esse contexto de mudanças sendo implantado em todo Brasil proporcionando novas ações e novas formas de organização dos serviços de saúde. ${ }^{25}$ Diante disso, entende-se que a enfermagem precisa acompanhar a proposta ministerial de mudança de modelo assistencial para atenção básica sem perder de vista o modelo assistencial da enfermagem baseado em cientificidade, ou seja, a partir da implantação do PE.

Devido ao grande número de estudos focalizando a área hospitalar como local de implantação da SAE fez-se necessário classificar os setores hospitalares onde as experiências se desenvolveram. Os setores com maior número de experiências de aplicação da SAE são as clínicas médica e obstetrícia, 28\%(20) e 13\%(9) respectivamente. 0 setor da 


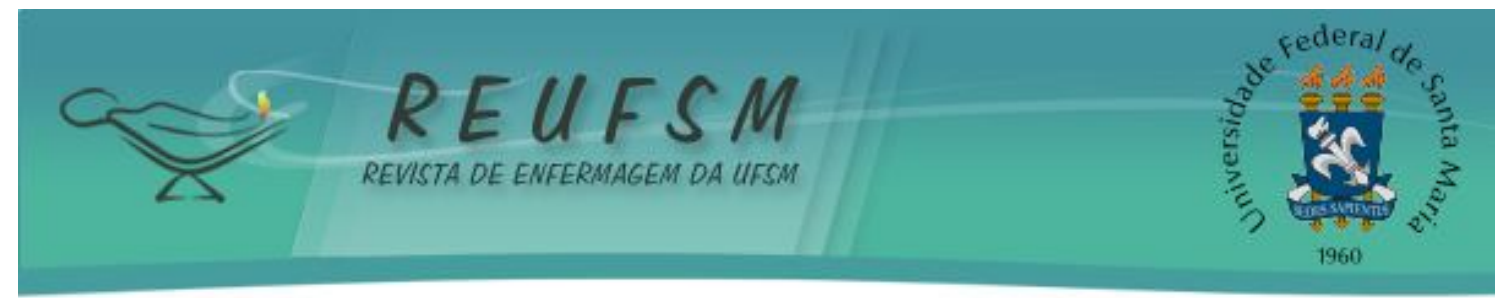

cardiologia aparece com um quantitativo de 6 estudos (8\%), seguido dos setores de clínica cirúrgica 7\%(5), pediatria 7\%(5) e pneumologia 6\%(4). Os setores da geriatria, nefrologia e a neurologia apresentaram uma porcentagem de $4 \%$ (3) nos estudos, seguido dos setores da dermatologia, oncologia, psiquiatria e terapia intensiva com 3\% (2). Ainda em menor quantitativo aparecem os estudos na endocrinologia, ortopedia, reumatologia, transplante e no transporte aéreo com a representatividade de 1\% (1). Estes resultados demonstram que não se pode traçar tendências para setores específicos, ao que parece, a real implantação do PE está mais ligada a consciência do enfermeiro no que diz respeito a metodologia de assistência sistematizada do que com a especificidade propriamente dita.

\section{Teorias de Enfermagem predominantes nos estudos}

O suporte teórico mais utilizado para orientar as etapas do $\mathrm{PE}$, nas experiências encontradas, foi a Teoria das Necessidades Humanas Basicas (NHB) de Wanda de Aguiar Horta sendo identificada em 36\% (26) dos trabalhos publicados. A teoria do Déficit do Autocuidado de Dorothea Orem também se destacou nos estudos analisados, mostrando-se presente em 12 (17\%) dos 71 trabal hos estudados. As demais publicações variaram entre os seguintes teóricos: Levine 3\%(2), Watson 3\%(2), Ida J ean Orlando 1\%(1), Irving 1\%(1), J oyce Travelbee 1\%(1), King 1\%(1), Neuman 1\%(1), Rawlins e Heacock 1\% (1), Roper Logan Tierney $1 \%(1)$, Wiedenbach $1 \%(1)$.

Chama a atenção o fato de que, embora $29 \%$ (21) dos estudos afirmarem a aplicação do PE não descrevem no seu resumo o referencial teórico utilizado para essa prática. Talvez essa ausência possa ser justificada por falta de informações que poderiam estar contidas no artigo completo, que por sua vez não estavam disponíveis para proceder a sua leitura na íntegra, limitando a interpretação dos autores no tocante a aplicação do PE sem o embasamento teórico.

As teorias podem ser definidas como uma conceituação articulada e comunicada da realidade, criada ou descoberta, dentro da enfermagem ou pertinentes a ela, para 0 propósito de descrição, explicação, predição ou prescrição do cuidado de enfermagem. Sendo assim, entende-se que não há possibilidade da aplicação da SAE sem um referencial teórico, mesmo porque, além de se perder o direcionamento das ações para atingir os objetivos traçados, se descumpriria a legislação vigente que reza pela necessidade iminente de utilização de um teórico de enfermagem para aplicar o modelo assistencial permeado pelo PE. ${ }^{26}$ Devendo ressaltar-se que as teorias de enfermagem constituem a base para nortear a prática cuidativa do enfermeiro e estabelecem as bases para a construção da enfermagem como ciência. ${ }^{27}$

Ao escolher uma teoria para fundamentar a sua prática profissional, o enfermeiro necessita antes: identificar as características estruturais e funcionais da unidade de saúde em que se pretende implementar a SAE, bem como conhecer o perfil dos enfermeiros que trabalham naquela unidade e 0 perfil dos pacientes atendidos. ${ }^{2}$ Para que todo processo seja efetivo é indispensável que os conceitos da teoria escol hida tenham congruência com as características mencionadas anteriormente.

Como dito antes, os trabalhos el encados para esse estudo citam prioritariamente duas teóricas de enfermagem, Wanda Horta e Dorothea Orem, sem necessariamente terem sido utilizadas em setores semelhantes. 0 questionamento que emerge desses achados, e sem a pretensão de respostas imediatas é: "Porque priorizar duas teóricas para aplicação de SAE em detrimento de tantas outras já conhecidas e respeitadas?". Embora não se tenha essa resposta, surge uma reflexão no sentido de se buscar compreender quais os mecanismos estão sendo utilizados pelos enfermeiros para escolher a teoria que irá embasar sua assistência. Acredita-se que pela dificuldade inerente ao processo de 


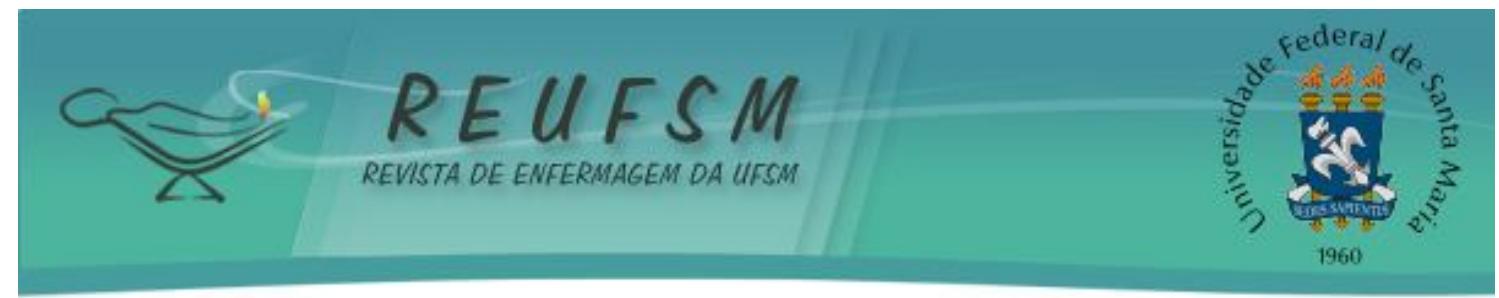

associação entre teorias e metodologia assistencial de enfermagem, há uma tendência natural de se utilizar teóricas que, embora não se conheça profundamente, são mais divulgadas nas universidades. Contudo, essa é apenas uma suposição, o alcance dessa resposta merece uma longa e profunda investigação.

Ao terminar essa revisão sistematizada e, resgatando a principal teórica representante do nosso país, uma sensação muito inquietante de lentidão no avanço da enfermagem se abate sobre os autores. Em 1979, no prefácio da 1a edição do seu Livro, Wanda Horta, relata "Com o processo de enfermagem a profissão atingiu sua maioridade... No Brasil ainda estamos nas fases iniciais da implantação do processo e esse fato se deve à insuficiente literatura sobre o assunto." Embora essa seja uma citação de 32 anos atrás, ao se realizar uma revisão de publicações sobre o mesmo tema nos dias atuais, fica a sensação de que não se avançou o suficiente. Ainda estamos caminhando a passos muito lentos na direção da plenitude da profissão do enfermeiro. Mesmo frente à obrigatoriedade legal da implantação do PE, grande parte dos enfermeiros no Brasil ainda não absorveram a SAE como única forma legal de se assistir o paciente.

\section{CONSIDERAÇÕES FINAIS}

Neste estudo evidenciaram-se várias temáticas relacionadas à $\mathrm{SAE}$, mas, sobretudo o desenvolvimento de estudos de caso, a elaboração de instrumentos de coleta, o levantamento do perfil diagnóstico de um setor e finalmente as experiências onde se aplicou, mesmo que parcialmente, o PE. No entanto, esta diversidade de temáticas não traduz o grande objetivo da SAE que é a aplicação do PE com todas as suas cinco fases interrelacionadas. Ao contrário, o estudo revelou que são poucas as experiências publicadas onde o PE é empregado de forma plena, com todas as fases, nas instituições de saúde.

Outro achado preocupante é o fato de que praticamente as experiências de sistematização concentram-se nos níveis secundários e terciários, sobretudo na área hospitalar. 0 nível primário de assistência à saúde quase não tem sido contemplado com experiências de SAE no levantamento realizado. Em um período da história da saúde brasileira onde os olhares estão voltados para a atenção básica, esta situação é alarmante. Ainda, embora se tenha constatado que não há uma tendência predominante para implantação da SAE em setores específicos, há um predomínio de utilização das teóricas Wanda Horta e Dorothea Orem.

Por fim entende-se que, ou os enfermeiros se mobilizam e promovem uma ação entre profissionais da área, pesquisadores, associações, órgãos legisladores e gestores a fim de legitimar a aplicação da SAE como metodologia assistencial de enfermagem única para um cuidado eficaz balizado na cientificidade ou regredimos à condição de acreditar que o PE nada mais é do que uma forma metodológica de organizar dados não focados no cuidado ao paciente.

\section{REFERÊNCIAS}

1. Gonçalves, AMP. Perfil diagnóstico de enfermagem admissional de pacientes com síndrome coronariana aguda. Belo Horizonte: [s.n.], 2004. 119 p. Dissertação (Mestrado em Enfermagem) - Universidade Federal de Minas Gerais, 2004.

2. Tannure MC, Pinheiro AM. SAE - Sistematização da Assistência de Enfermagem: guia prático. Meire Chucre Tannure, Ana Maria Pinheiro. 2a ed. - Rio de Janeiro: Guanabara Koogan, 2010. 298 p. 


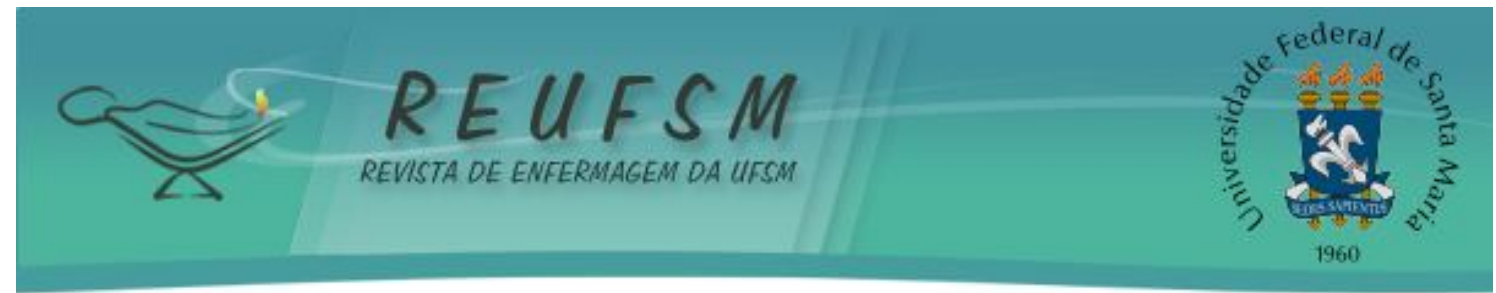

3. Conselho Federal de Enfermagem. Resolução COFEN n॰ 358 de 15 de outubro de 2009. Dispõe sobre a Sistematização da Assistência de Enfermagem - SAE - nas Instituições de Saúde Brasileiras. Rio de J aneiro: Conselho Federal de Enfermagem; 2009.

4. Nascimento KC, Backes DS, Koerich MS, Erdmann AL. Sistematização da assistência de enfermagem: vislumbrando um cuidado interativo, complementar e multiprofissional. Rev Esc Enferm USP. 2008; 42(4): 643-648.

5. Santos, SR, Nóbrega, MML. A busca da interação teórica e prática no sistema de informação em enfermagem - enfoque na teoria fundamentada nos dados. Rev Latinoam enferm. 2004; 12(3): 460-468.

6. Kletemberg DF, Siqueira MD, Mantovani Maria de Fátima. Uma história do processo de enfermagem nas publicações da Revista Brasileira de Enfermagem no período 1960-1986. Esc Anna Nery Rev Enferm. 2006; 10(3): 478-486.

7. Gaidzinski RR, Soares AVN, Lima AFC, Gutierrez BAO, Cruz DALM, Rogenski NMB, Sancinetti TR. Diagnóstico de enfermagem na prática clínica. In: Cruz DALM. Processo de enfermagem e classificações. Porto Alegre: Artmed 2008. p. 25-37.

8. Gaidzinski RR, Soares AVN, Lima AFC, Gutierrez BAO, Cruz DALM, Rogenski NMB, Sancinetti TR. Diagnóstico de enfermagem na prática clínica. In: Cerullo J ASB, Cruz DALM. Implementação dos diagnósticos de enfermagem da NANDA-I em hospitais brasileiros. Porto Alegre: Artmed 2008. p.38-46.

9. Conselho Federal de Enfermagem (COFEN). Resolução n. 272, de 27 de agosto de 2002: Dispõe sobre a Sistematização da Asistência de Enfermagem - SAE. Brasília: 2002. [acesso 2008 mar. 12]. Disponível em: http// www. portalcofen.gov.br

10. Cunha SMB, Barros ALBL. Análise da implementação da Sistematização da Assistência de Enfermagem, segundo o Modelo Conceitual de Horta. Rev bras enferm. 2005; 58(5): 568-572.

11. Pereira J C, Stuchi RAG, Arreguy-Sem C. Proposta de sistematização da assistência de enfermagem pelas taxonomias nanda/nic/noc para 0 diagnostico de conhecimento deficiente. Cogitare enferm. 2010; 15(1):74-81.

12. Pellicia R, Moritaka L, Paegle LD, Roncaratti E, Pisetta V, Sassaki MM, Alves PRA. Sistematizaçäo da assistência de enfermagem ao colostomizado ileostomizado em um hospital privado. Rev paul enferm. 1992; 11(1):41-5.

13. Truppel TC, Meier MJ , Calixto RC, Peruzzo SA, Crozeta K. Sistematização da Assistência de Enfermagem em Unidade de Terapia Intensiva. Rev bras enferm. 2009; 62(2): 221-227.

14. Freire MRSM, Nóbrega MML. O idoso hipertenso e o autocuidado. Rev RENE. 2001; 2(1): 60-68.

15. Margarido ES, Castilho V. Aferição do tempo e do custo médio do trabalho da enfermeira na consulta de enfermagem. Rev Esc Enferm USP. 2006; 40(3): 427-433.

16. Bittar DB, Pereira LV, Lemos RCA. Sistematização da assistência de enfermagem ao paciente crítico: proposta de instrumento de coleta de dados. Texto \& contexto enferm. 2006; 15(4): 617-628.

17. Lima GS, Cavalcante TMC, Isabella APJ, Magalhães AS. Assistência de enfermagem a um paciente infartado portador de HIV, baseada na teoria do autocuidado: estudo de caso. Acta paul. enferm. 2007; 20(4): 452-457. 


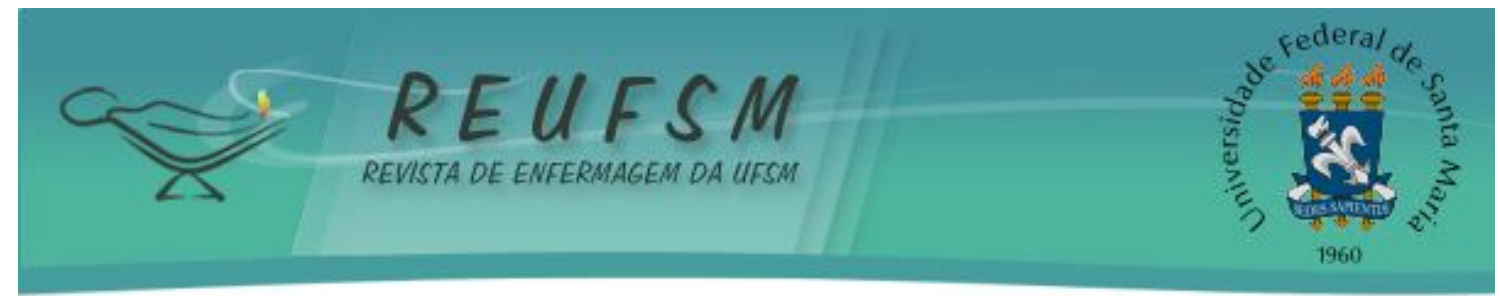

18. Herbas PJ S. Avaliação da contribuição do sistema informatizado em enfermagem para o enfermeiro e sua aplicabilidade no ponto de cuidado do paciente. São Paulo: [s.n], 2009. 207 p. Tese (Doutorado) - Faculdade de Medicina da Universidade de São Paulo, 2009.

19. Silva MR. Principais diagnósticos de enfermagem em pacientes portadores da síndrome da imunodeficiência adquirida(AIDS). São Paulo: [s.n], 2005. 118 p. Dissertação (Mestrado) - Universidade Federal de São Paulo. Escola Paulista de Medicina, 2005.

20. Luvisotto MM , Carvalho R, Galdeano LE. Transplante renal: diagnósticos e intervenções de enfermagem de pacientes no pós-operatórioimediato. Einstein. São Paulo: 2007; 5(2): 117-122.

21. Horta WA. Processo de enfermagem. São Paulo: EPU, 1979.

22. Siqueira RC. Diagnósticos de enfermagem em crianças menores de um ano acompanhadas na consulta de enfermagem em puericultura numa unidade básica de saúde de Fortaleza-CE. Fortaleza: [s.n.], 2005. 107 p. Dissertação (Mestrado) - Universidade Federal do Ceará, 2005.

23. Dell'Acqua MCQ; Miyadahira AMK. Processo de enfermagem: fatores que dificultam e os que facilitam o ensino. Rev Esc Enferm USP. 2000; 34(4): 383-389.

24. Carvalho EC, Bachion MM, Dalri MCB, J esus CAC. Obstáculos para a implementação do processo de enfermagem no Brasil. Rev enferm UFPE on line [REUOL]. [periódico na Internet]. 2007 [citado 2011 Mar 15];1(1). Disponível em: http:// www. ufpe. br/ revistaenfermagem/ index. php/ revista/article/ viewArticle/ 17. doi: 10.5205/ 01012007.

25. Camargo ATSP. Regionalização da saúde face à realidade metropolitana: análise das internações hospitalares na região metropolitana do Rio de Janeiro (1995-2005). Rio de J aneiro: [s.n.], 2009. 149 p. Tese (Doutorado) - Universidade do Estado do Rio de J aneiro. Instituto de Medicina Social, 2009.

26. Leopardi MT. Teorias em Enfermagem - instrumentos para a prática. Florianópolis: Papa Livros, 1999.

27. George JB (coord.) et al. Teorias de enfermagem. Dos Fundamentos à Prática Profissional . 4ạ ed. Porto Alegre: Artes Médicas, 2000.

Data de recebimento: 13/04/2011

Data de aceite: 29/06/2011

Contato com autor responsável: Ricardo Bezerra Cavalcante

Endereço postal: Universidade Federal São J oão Del Rei - UFSJ

Campos Centro Oeste Dona Lindu, Av. Sebastião Gonçalves Coelho no 400, Chanadour.

Divinópolis/MG.

CEP: 35504-296.

E-mail: ricardocaval canteufmg@yahoo.com.br 